\title{
A simple, noninvasively determined index predicting hepatic failure following liver resection for hepatocellular carcinoma
}

\author{
Tsuyoshi Ichikawa $\cdot$ Takahiro Uenishi $\cdot$ Shigekazu Takemura $\cdot$ Kazuki Oba $\cdot$ Masao Ogawa $\cdot$ \\ Shintaro Kodai · Hiroji Shinkawa - Hiromu Tanaka - Takatsugu Yamamoto - Shogo Tanaka • \\ Satoshi Yamamoto $\cdot$ Seikan Hai $\cdot$ Taichi Shuto $\cdot$ Kazuhiro Hirohashi $\cdot$ Shoji Kubo
}

Received: 15 October 2007/Accepted: 16 January 2008/Published online: 13 December 2008

(C) Springer 2008

\begin{abstract}
Background A novel index, the serum aspartate aminotransferase activity/platelet count ratio index (APRI), has been identified as a biochemical surrogate for histological fibrogenesis and fibrosis in cirrhosis. We evaluated the ability of preoperative APRI to predict hepatic failure following liver resection for hepatocellular carcinoma.

Methods Potential preoperative risk factors for postoperative hepatic failure (hepatic coma with hyperbilirubinemia, four patients; intractable pleural effusion or ascites, 30 patients; and variceal bleeding, one patient) as well as APRI were evaluated in 366 patients undergoing liver resection for hepatocellular carcinoma. Prognostic significance was determined by univariate and multivariate analyses.

Results Hepatic failure developed postoperatively in 30 patients, causing death in four. APRI correlated with histological intensity of hepatitis activity and degree of hepatic fibrosis, and was significantly higher in patients who developed postoperative hepatic failure than in others without failure. Risk of postoperative hepatic failure increased as the serum albumin concentration and platelet count decreased and as indocyanine green retention rate at

T. Ichikawa $(\bowtie) \cdot$ S. Takemura $\cdot$ K. Oba $\cdot$ M. Ogawa $\cdot$

S. Kodai $\cdot$ H. Shinkawa $\cdot$ H. Tanaka .

S. Yamamoto $\cdot$ S. Hai $\cdot$ S. Kubo

Department of Hepato-Biliary-Pancreatic Surgery,

Osaka City University Graduate School of Medicine,

1-4-3 Asahimachi, Abeno-ku, Osaka 545-8585, Japan

e-mail: t-ichikawa@msic.med.osaka-cu.ac.jp

T. Uenishi $\cdot$ T. Yamamoto $\cdot$ S. Tanaka

Department of Surgery, Ishikiri-Seiki Hospital, Osaka, Japan

T. Shuto $\cdot$ K. Hirohashi

Department of General Practice,

Osaka City University Hospital, Osaka, Japan
\end{abstract}

15 min, aspartate and alanine aminotransferase activities, and APRI increased. Only APRI was an independent preoperative factor on multivariate analysis. Of the four patients who died of postoperative hepatic failure, three had an APRI of at least 10.

Conclusions Preoperative APRI independently predicted hepatic failure following liver resection for hepatocellular carcinoma. Patients with an APRI of 10 or more have a high risk of postoperative hepatic failure.

Keywords Liver resection - Hepatocellular carcinoma · Liver failure $\cdot$ APRI $\cdot$ Platelet count

$\begin{array}{ll}\text { Abbreviations } \\ \text { HCC } & \text { Hepatocellular carcinoma } \\ \text { APRI } & \begin{array}{l}\text { Aspartate aminotransferase/platelet count } \\ \text { ratio index }\end{array} \\ \text { Anti-HCV } & \text { Anti-hepatitis C virus antibody } \\ \text { HBsAg } & \text { Hepatitis B surface antigen } \\ \text { ICGR } 15 & \text { Indocyanine green retention rate at } 15 \text { min } \\ \text { AST } & \text { Aspartate aminotransferase } \\ \text { ALT } & \text { Alanine aminotransferase } \\ \text { AFP } & \alpha \text {-Fetoprotein } \\ \text { HAI } & \text { Histological activity index }\end{array}$

\section{Introduction}

Liver resection in patients with hepatocellular carcinoma (HCC) may result in postoperative hepatic failure, since most patients with HCC also have chronic liver disease including cirrhosis [1-9]. To avoid resection likely to lead to postoperative hepatic failure, various methods have been developed for preoperative assessment of liver function [4, 
5, 9-19]. However, some of these are complex and require calculations involving multiple formulas.

Previous studies have identified hepatic fibrosis and active hepatitis as negative predictive factors for effective liver regeneration and as risk factors for postoperative hepatic failure [3, 9, 20, 21]. Histopathologic examination of liver biopsy specimens presently remains the "gold standard" for assessing degree of active hepatitis and hepatic fibrosis. However, liver biopsy cannot be performed in all candidates for liver surgery because of its cost and risk of complications. Recently, a novel index, the aspartate aminotransferase (AST)/platelet count ratio index (APRI), has been identified as a biochemical marker for histological fibrogenesis and fibrosis in cirrhosis [22, 23]. APRI has been reported to correlate with hepatic fibrosis and magnitude of abnormalities in liver function tests [22, 23]. This simple ratio is calculated from results of routinely available, noninvasive, and inexpensive laboratory tests.

In the present study, we evaluated the relationship between preoperative APRI and postoperative hepatic failure following liver resection, and the ability of the ratio to serve as a marker to identify candidates who cannot safely undergo liver resection for HCC.

\section{Patients and methods}

\section{Patients and determination of APRI}

From the beginning of 1994 to the end of 2004, liver resection for HCC was performed for 372 patients in our department. Patients excluded from this study included five who underwent concomitant resection of a synchronous second tumor, and one who underwent liver resection combined with right atrial tumor thrombectomy requiring extracorporeal circulation. The 366 patients remaining as subjects in this study included 297 men and 69 women; their ages ranged from 30 to 85 years (mean $\pm \mathrm{SD}$, $63.6 \pm 8.2$ ). In total, 249 patients were positive for antihepatitis C antibody (anti-HCV) alone, 54 patients for hepatitis B surface antigen (HBsAg) alone, four patients for both anti-HCV and HBsAg, and 59 patients were negative for both viral markers. APRI was calculated as AST activity (IU/L)/platelet count $\left(10^{4} / \mu \mathrm{l}\right)[22]$.

The study was conducted in accordance with the Helsinki Declaration and the guidelines of the Ethics Committee at our institution.

Postoperative hepatic failure

Variables previously shown to predict postoperative hepatic failure included hepatic coma with hyperbilirubinemia (total serum bilirubin concentration $>5 \mathrm{mg} / \mathrm{dl}$ for more than
5 days); intractable pleural effusion or ascites requiring use of diuretics or thoracocentesis, or abdominal paracentesis on two or more occasions or institution of continuous abdominal drainage; or variceal bleeding [24, 25].

\section{Operative procedure}

The operative procedures is outlined below (major hepatectomy; segmentectomy or greater, minor hepatectomy; smaller than segmentectomy, anatomic hepatectomy; subsegmentectomy or greater, nonanatomic hepatectomy; smaller than subsegmentectomy).

\section{Pathologic examination}

Surgical specimens were cut serially into tissue blocks $5 \mathrm{~mm}$ thick, fixed in $10 \%$ formalin, and stained with hematoxylin and eosin. Histologic grade with respect to tumor differentiation was assigned using a modification of the classification by Edmondson and Steiner [26, 27]. The histologic activity index (HAI) was used with some modifications $[28,29]$ to evaluate severity of active hepatitis (histologic activity grade) and degree of hepatic fibrosis (histologic fibrosis grade). HAI scores consist of four components: component 1, periportal necrosis with or without bridging necrosis; component 2 , intralobular degeneration and focal necrosis; component 3 , portal inflammation; and component 4 , fibrosis. Summed HAI scores of 0 for (components 1-3) indicated no activity (histologic activity score, 0 ); scores of $1-3$, minimal activity (histologic activity score, 1 ); scores of 4-8, mild activity (histologic activity score, 2); scores of 9-12, moderate activity (histologic activity score, 3 ); and score of 13 or more indicated severe activity (histologic activity score, 4). The degree of fibrosis (histologic fibrosis score) was determined from component 4 of the HAI score. A histologic fibrosis grade of 1 indicated portal fibrous expansion; a grade of 2 indicated the presence of portalportal septa without architectural distortion; a grade of 3 indicated portocentral septa with architectural distortion; and a grade of 4 indicated cirrhosis.

\section{Statistics}

Student's $t$ test was used to examine differences in age and tumor size. The Mann-Whitney $U$ test was used to examine differences in laboratory test results. Fisher's exact test or the $\chi^{2}$ test was used to compare categorical data between groups. Correlations between APRI and results of other laboratory tests were determined using Pearson's correlation coefficient. The correlation between the APRI and histologic activity score or fibrosis score in noncancerous liver was determined by Spearman's rank correlation. Odds ratios were used to estimate relative risk for postoperative hepatic failure. 
Logistic regression was used for univariate analysis, while multiple logistic regression analysis was used for multivariate analysis. For multivariate analysis, variables possibly significant $(P<0.1)$ on univariate analysis were evaluated. $P$ values less than 0.05 were considered significant.

Table 1 Correlations between APRI and results of other laboratory tests

\begin{tabular}{lcr}
\hline Laboratory test & Correlation coefficient & $P$ value \\
\hline Total bilirubin & 0.306 & $<0.0001$ \\
Albumin & -0.248 & $<0.0001$ \\
ICGR $_{15}$ & 0.280 & $<0.0001$ \\
AST & 0.756 & $<0.0001$ \\
ALT & 0.625 & $<0.0001$ \\
Platelet count & -0.555 & $<0.0001$ \\
Prothrombin test $(\%)$ & -0.132 & 0.0119 \\
\hline
\end{tabular}

$I C G R_{15}$ Indocyanine green retention rate at $15 \mathrm{~min}, A S T$ aspartate aminotransferase, $A L T$ alanine aminotransferase

\section{Results}

Preoperative APRI, which ranged from 0.6 to 51.3 (mean $\pm \mathrm{SD}, 5.0 \pm 4.4$ ), correlated with serum concentrations of total bilirubin and albumin, indocyanine green retention test results at $15 \mathrm{~min}\left(\mathrm{ICGR}_{15}\right)$, serum activities of AST and alanine aminotransferase (ALT), platelet count, and prothrombin test (Table 1).

APRI also correlated with both histologic activity score $(P=0.0003)$ and histologic fibrosis score $(P=0.0247)$.

Postoperative hepatic failure developed in 30 patients (hepatic coma with hyperbilirubinemia in four patients; intractable pleural effusion or ascites in 30 patients; and variceal bleeding in one patient). Four of these patients died of hepatic failure while still in the hospital. Clinicopathologic findings were compared between the 30 patients with postoperative hepatic failure and the 336 patients without failure (Table 2). These groups were similar in age, gender, percentage of patients with a history of alcohol abuse (defined as an estimated daily intake of $86 \mathrm{~g}$ of
Table 2 Demographic and clinicopathologic features of patients with and without hepatic failure after liver resection
Most laboratory results, intraoperative blood loss, and time of operation are given as medians (with 10th and 90th percentiles)

Anti-HCV Anti-hepatitis C virus antibody, HBsAg hepatitis B surface antigen, $I C G R_{15}$ indocyanine green retention rate at $15 \mathrm{~min}, A S T$ aspartate aminotransferase, $A L T$ alanine aminotransferase, $A F P \alpha$ fetoprotein

\begin{tabular}{|c|c|c|c|}
\hline \multirow[t]{2}{*}{ Parameter } & \multicolumn{2}{|l|}{ Hepatic failure } & \multirow[t]{2}{*}{$P$ value } \\
\hline & No, $n=336$ & Yes, $n=30$ & \\
\hline Age, years, mean $\pm S D$ & $63.6 \pm 8.4$ & $64.0 \pm 6.9$ & 0.8912 \\
\hline Gender, M:F & $274: 62$ & $23: 7$ & 0.5125 \\
\hline Alcohol abuse & 115 & 10 & 0.9593 \\
\hline History of blood transfusion & 75 & 8 & 0.5922 \\
\hline Anti-HCV & 229 & 24 & 0.1784 \\
\hline HBs-Ag & 54 & 4 & 0.6940 \\
\hline Diabetes mellitus & 55 & 7 & 0.4562 \\
\hline Total bilirubin (mg/dl) & $0.8(0.5,1.3)$ & $0.9(0.5,1.4)$ & 0.3008 \\
\hline Albumin $(\mathrm{g} / \mathrm{dl})$ & $3.7(3.3,4.2)$ & $3.6(2.9,4.1)$ & 0.0309 \\
\hline $\mathrm{ICGR}_{15}(\%)$ & $15.0(7.5,25.2)$ & $17.8(7.0,34.5)$ & 0.2434 \\
\hline AST (IU/l) & $52(30,96)$ & $64(46,107)$ & 0.0208 \\
\hline ALT (IU/l) & $56(26,113)$ & $70(38,129)$ & 0.0232 \\
\hline Platelet count $\left(\times 10^{4} / \mu \mathrm{l}\right)$ & $14.3(8.4,22.9)$ & $11.6(5.6,20.5)$ & 0.0068 \\
\hline Prothrombin test $(\%)$ & $94(73,135)$ & $94(76,131)$ & 0.9798 \\
\hline APRI & $3.8(1.6,9.0)$ & $5.3(2.6,13.3)$ & 0.0039 \\
\hline AFP, $>20 \mathrm{ng} / \mathrm{ml}$ & 180 & 18 & 0.5200 \\
\hline Tumor size, $\mathrm{cm}$, mean $\pm \mathrm{SD}$ & $3.8 \pm 2.8$ & $4.2 \pm 2.8$ & 0.4504 \\
\hline Operative procedure, major:minor & $125: 211$ & $14: 16$ & 0.3061 \\
\hline Operative procedure, anatomic:nonanatomic & 170:166 & $17: 13$ & 0.2571 \\
\hline Intraoperative blood loss $(\mathrm{g})$ & $725(196,2595)$ & $1450(478,3662)$ & 0.0002 \\
\hline Time of operation (min) & $282(180,440)$ & $317(213,500)$ & 0.1016 \\
\hline \multicolumn{4}{|l|}{ Histologic activity grade } \\
\hline $0-2$ & 300 & 20 & 0.0003 \\
\hline 3 & 34 & 10 & \\
\hline \multicolumn{4}{|l|}{ Histologic fibrosis grade } \\
\hline $0-3$ & 194 & 11 & 0.0247 \\
\hline 4 , representing cirrhosis & 141 & 19 & \\
\hline
\end{tabular}




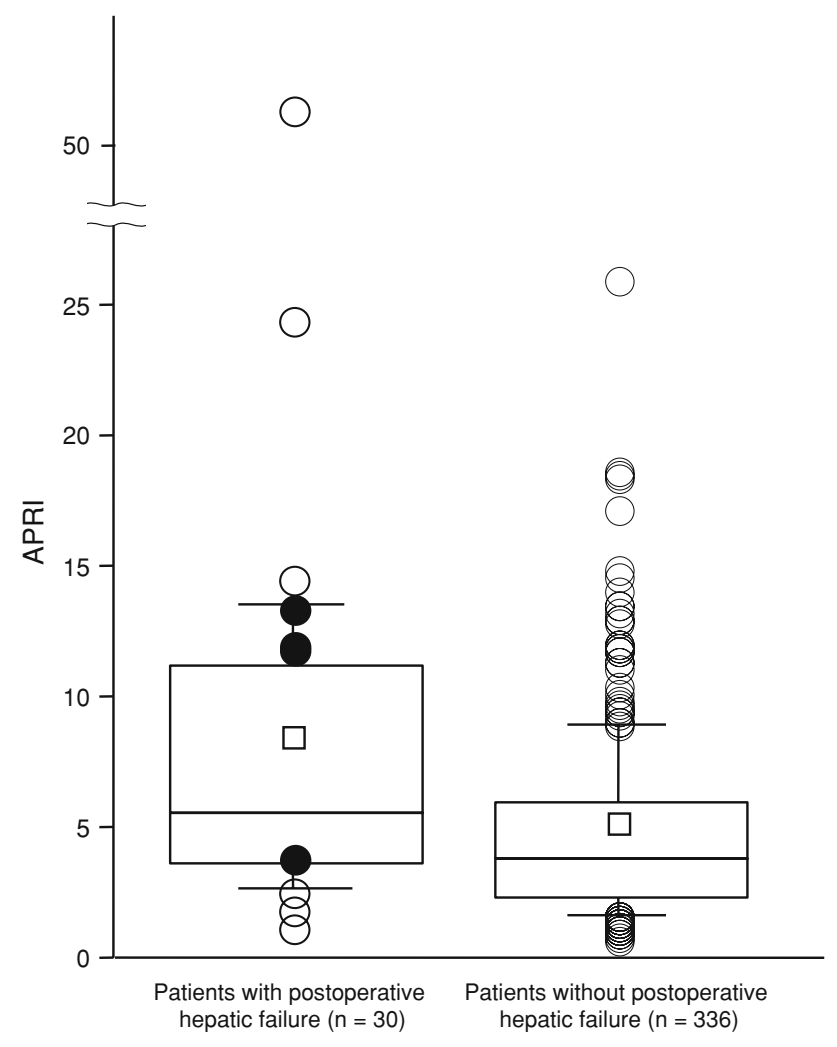

Fig. 1 APRI in two groups of patients who underwent liver resection for hepatocellular carcinoma. APRI values of patients with and without postoperative hepatic failure ranged from 0.6 to 26 and from 1.1 to 51.3 , respectively (mean $\pm \mathrm{SD}$, means shown as small squares, $8.3 \pm 9.3$ and $4.7 \pm 3.5$ ). Bars, 10th and 90th percentiles; bottoms and tops of boxes, 25th and 75th percentiles; lines across boxes, medians; circles, outliers; closed circles, patients who died of postoperative hepatic failure

ethanol for at least 10 years, according to the criteria of the Liver Cancer Study Group of Japan [30]), diabetes mellitus, blood transfusion, and anti-HCV and $\mathrm{HBsAg}$ positivity. Although serum total bilirubin concentration, ICGR $_{15}$, and prothrombin test results did not differ between groups, serum albumin concentration and platelet count were significantly lower in patients with postoperative hepatic failure than in these without it. Serum activities of AST and ALT and also the APRI (Fig. 1) were significantly higher in patients with than without postoperative hepatic failure. Although no difference was noted in tumor size, operative procedure, or operative time was noted between them, intraoperative blood loss was significantly greater in patients who developed postoperative hepatic failure. The percentage of patients with moderate hepatitis activity (histologic activity grade 3 ) or cirrhosis (histologic fibrosis grade 4) was significantly greater in patients who developed postoperative hepatic failure.

Table 3 shows odds ratios (OR) for the candidate risk factors associated with postoperative hepatic failure as
Table 3 Risk factors for hepatic failure after liver resection for hepatocellular carcinoma, evaluated by univariate analysis

\begin{tabular}{llll}
\hline Variable & $\begin{array}{l}\text { Odds } \\
\text { ratio }\end{array}$ & $95 \%$ CI & $P$ value \\
\hline $\begin{array}{l}\text { Age (per 1 year) } \\
\text { Gender }\end{array}$ & 1.003 & $0.958-1.050$ & 0.8908 \\
Female & & & \\
Male & 1.000 & & \\
Alcohol abuse & 0.743 & $0.305-1.810$ & 0.5138 \\
$(-)$ & & & \\
$(+)$ & 1.000 & & \\
History of blood transfusion & 0.979 & $0.441-2.177$ & 0.9593 \\
$(-)$ & & & \\
$(+)$ & 1.000 & & \\
Anti-HCV & 1.261 & $0.539-2.947$ & 0.5929 \\
$(-)$ & & & \\
$(+)$ & 1.000 & & \\
HBsAg & 1.869 & $0.742-4.707$ & 0.1844 \\
$(-)$ & & & \\
$(+)$ & 1.000 & & \\
(+) & 0.803 & $0.270-2.395$ & 0.6945
\end{tabular}

Diabetes mellitus

$(-)$

1.000

$(+)$

$1.406 \quad 0.572-3.456 \quad 0.4580$

AFP (ng/ml)

$\leq 20$

1.000

$>20$

$1.283 \quad 0.599-2.748 \quad 0.5208$

Total bilirubin (per $1 \mathrm{mg} / \mathrm{ml}$ )

$1.788 \quad 0.616-5.184 \quad 0.2848$

Albumin (per $1 \mathrm{~g} / \mathrm{dl}$ )

$\begin{array}{lll}0.241 & 0.086-0.678 & 0.0070\end{array}$

ICGR $_{15}($ per 1\%)

$\begin{array}{lll}1.046 & 1.005-1.088 & 0.0272\end{array}$

AST (per $1 \mathrm{IU} / \mathrm{l}$ )

$\begin{array}{lll}1.008 & 1.000-1.017 & 0.0459\end{array}$

ALT (per 1 IU/l)

$\begin{array}{lll}1.008 & 1.000-1.016 & 0.0572\end{array}$

Platelet count (per $\left.1 \times 10^{4} / \mu \mathrm{l}\right)$

$\begin{array}{lll}0.925 & 0.860-0.996 & 0.0385\end{array}$

Prothrombin test (per 1\%)

APRI (per 1)

$0.999 \quad 0.983-1.016 \quad 0.9279$

$1.125 \quad 1.045-1.211 \quad 0.0017$

Operative procedure

Minor $\quad 1.000$

$\begin{array}{llll}\text { Major } & 1.477 & 0.697-3.129 & 0.3085\end{array}$

Operative procedure

Non-anatomic 1.000

Anatomic 1.529

$0.736-3.175 \quad 0.2545$

Intraoperative blood loss (per $1 \mathrm{~g}$ )

$\begin{array}{lll}1.000 & 1.000-1.001 & 0.0147\end{array}$

Time of operation (per $1 \mathrm{~min}$ )

$1.003 \quad 1.000-1.006 \quad 0.0804$

Histologic activity grade

\begin{tabular}{llll}
$0-2$ & 1.000 & & \\
3 & 4.286 & $1.858-9.888$ & 0.0006 \\
Histologic fibrosis grade & & & \\
$1-3$ & 1.000 & & \\
4, representing cirrhosis & 2.377 & $1.096-5.152$ & 0.0283 \\
\hline
\end{tabular}

Anti-HCV Anti-hepatitis C virus antibody, $H B s A g$ hepatitis B surface antigen, $I C G R_{15}$ indocyanine green retention rate at $15 \mathrm{~min}, A S T$ aspartate aminotransferase, $A L T$ alanine aminotransferase, $A F P \alpha$-fetoprotein 
calculated by univariate analysis. Serum albumin concentration $\quad(\mathrm{OR}=0.241), \quad \mathrm{ICGR}_{15} \quad(\mathrm{OR}=1.046), \quad \mathrm{AST}$ $(\mathrm{OR}=1.008), \quad$ ALT $\quad(\mathrm{OR}=1.008), \quad$ platelet $\quad$ count $(\mathrm{OR}=0.925)$, APRI $(\mathrm{OR}=1.125)$, histologic activity grade $3(\mathrm{OR}=4.286)$, and cirrhosis $(\mathrm{OR}=2.377)$ were risk factors for postoperative hepatic failure. APRI in three of the four patients who died of postoperative hepatic failure was 10 or more. Risk of postoperative hepatic failure increased as serum albumin concentration and platelet count decreased, and as ICGR $_{15}$, AST, and ALT activities and APRI increased. Risk of postoperative hepatic failure also increased with greater intraoperative blood loss, and was also high in patients with moderately active hepatitis or cirrhosis according to histopathologic examination. Risk of postoperative hepatic failure correlated closely with both severity of active hepatitis and degree of hepatic fibrosis.

Multivariate analysis was used to estimate the adjusted odds ratio for postoperative hepatic failure based on preoperative data (Table 4). Because the aim of this study was to evaluate the role of the APRI as preoperative prediction

Table 4 Risk factors for hepatic failure after liver resection for hepatocellular carcinoma, evaluated by multivariate analysis

\begin{tabular}{llll}
\hline Variable & Odds ratio & $95 \%$ CI & $P$ value \\
\hline Albumin (per 1 g/dl) & 0.360 & $0.114-1.140$ & 0.0823 \\
ICGR $_{15}$ (per 1\%) & 1.026 & $0.975-1.081$ & 0.3222 \\
Prothrombin test (per 1\%) & 1.007 & $0.990-1.025$ & 0.4052 \\
APRI & 1.098 & $1.018-1.184$ & 0.0149 \\
\hline
\end{tabular}

$I C G R_{15}$ Indocyanine green retention rate at $15 \mathrm{~min}, A P R I$ AST to platelet ratio index

Table 5 Features of hepatic failure in patients with high or low APRI

\begin{tabular}{lccr}
\hline Feature & \multicolumn{2}{l}{ APRI } & \multirow{2}{*}{$P$ value } \\
\cline { 2 - 3 } & $\begin{array}{l}\leq 10 \\
(n=333)\end{array}$ & $\begin{array}{l}>10 \\
(n=33)\end{array}$ \\
\hline Hepatic coma & 2 & 2 & 0.0422 \\
Intractable pleural effusion or ascites & 23 & 10 & $<0.0001$ \\
Variceal bleeding & 0 & 1 & 0.0902 \\
Death from hepatic failure & 1 & 3 & 0.0025 \\
\hline
\end{tabular}

of postoperative hepatic failure; significant risk factors on univariate analysis which were acquired postoperatively were eliminated. APRI was the only independent preoperative risk factor for postoperative hepatic failure (adjusted OR $=1.098,95 \%$ CI; 1.018-1.184).

We next compared the 330 patients with a low APRI $(<10)$ with the 33 patients whose APRI was high $(\geq 10)$ (Table 5). A cut-off line for APRI of 10 yielded the most significant differences in cut off lines (counting number) which we decided. The sensitivity, specificity, and positive predictive value of APRI $\geq 10$ for postoperative hepatic failure were $27.3 \%, 7.8 \%$, and $27.3 \%$, respectively. The prevalence of hepatic coma and intractable pleural effusion or ascites was significantly higher among patients with a high APRI than in those with a low APRI. Variceal bleeding developed in only one patient, who had an elevated APRI $(\geq 10)$. Although only 1 of the 333 patients with a low APRI died of postoperative hepatic failure, 3 of 33 patients with a high APRI died of this complication $(P=0.0025)$. APRI was $\geq 10$ in three of four patients who died of postoperative hepatic failure. The serum concentrations of total bilirubin and albumin, $\mathrm{ICGR}_{15}$, and prothrombin test results were distributed over wide ranges in these four patients (Table 6).

The clinical significance of the APRI in the determination of operative methods was investigated. The APRI was 10 or greater in only 4 of 139 patients who underwent major hepatectomy; it is thus not possible to evaluate the role of APRI in only patients who underwent major hepatectomy. For the 227 patients who underwent minor hepatectomy, APRI was a significant risk factor for postoperative hepatic failure.

\section{Discussion}

In this study, we attempted to develop a preoperative risk marker for predicting postoperative hepatic failure in patients with hepatocellular carcinoma who are candidates for hepatic resection using only routine laboratory tests. High APRI was a risk factor for postoperative hepatic failure on univariate analysis and the only independent preoperative risk factor on multivariate analysis.

Table 6 Results of laboratory tests and operative methods in four patients who died of postoperative hepatic failure

\begin{tabular}{llllllll}
\hline Patient no. & Age (year) & T-bil $(\mathrm{mg} / \mathrm{dl})$ & Albumin $(\mathrm{g} / \mathrm{dl})$ & ICGR $_{15}(\%)$ & Prothrombin test $(\%)$ & APRI & Operative method \\
\hline 1 & 72 & 0.3 & 3.0 & 37.4 & 74 & 11.0 & Partial \\
2 & 62 & 0.9 & 3.0 & 13.0 & 94 & 12.7 & Partial \\
3 & 59 & 1.4 & 3.8 & 23.1 & 100 & 3.96 & Rt. lobectomy \\
4 & 55 & 1.3 & 2.9 & 26.9 & 104 & 11.5 & Partial \\
\hline
\end{tabular}

T-bil Total bilirubin, $I C G R_{15}$ indocyanine green retention rate at $15 \mathrm{~min}, A P R I$ AST to platelet ratio index 
Cirrhosis is a well-known risk factor for postoperative hepatic failure [3, 10, 13, 20, 31, 32]. Degree of hepatic fibrosis is a negative predictor of liver regeneration and restoration of liver function after liver resection [33] that also influences the risk of postoperative ascites and pleural effusion [34] and duration of postoperative hepatic failure [9]. Active hepatitis has also been reported to be a potential risk factor for hepatic failure following liver resection [9, $31,35]$. We previously reported that active hepatitis and severe hepatic fibrosis are each risk factors for postoperative hepatic failure [25]. However, since biopsy specimens of noncancerous hepatic tissue are not readily obtainable preoperatively from all patients for reasons of safety and other issues, less invasive markers are needed. Recently, the APRI, which is calculated from AST activity and the platelet count, has been reported to correlate with histologic degree of hepatic fibrosis [22, 23]. In the present study we confirmed that APRI correlated with both histologic activity grade and histologic fibrosis grade in patients with HCC. Thus, preoperative determination of the APRI informs the surgeon about severity of active hepatitis and degree of fibrosis, and provides a measure of the risk of postoperative hepatic failure.

The indocyanine green clearance test has been used to develop guidelines for extent of liver resection [3, 5, 7, 18, 21, 36-42]. In this study, the role of the APRI in decisionmaking concerning extent of liver resection since remained unclear. In fact, it was not possible to evaluate the role of the APRI, since the APRI was 10 or greater in only 4 of 139 patients who underwent major hepatectomy. The percentage of patients with postoperative hepatic failure was significantly higher among those with a high APRI $(\geq 10)$ than among those with a low APRI $(<10)$. The percentage of patients who died of hepatic failure also was significantly higher among those with a high APRI than among those with a low APRI. We suggest that APRI $\geq 10$ is a more useful marker for postoperative death than any other laboratory tests (total bilirubin, albumin, $\mathrm{ICGR}_{15}$, and prothrombin test). The APRI was thus useful for determining whether liver resection itself is indicated in patients with chronic liver disease. Since patients who have a high APRI have active hepatitis and severe hepatic fibrosis, care is needed to avoid postoperative hepatic failure. Treatments for HCC include locoregional treatment including percutaneous ethanol injection, microwave coagulation therapy, and radiofrequency ablation therapy and transarterial therapy, including transcatheter arterial embolization and hepatic arterial infusion chemotherapy [43]. These alternatives can be considered for patients with a high APRI.

Other reported risk factors for postoperative hepatic failure include patient age [10,31], diabetes mellitus [13], and excessive intraoperative blood loss $[7,13,18,21,32$, 33]. Although excessive intraoperative blood loss was a risk factor on univariate analysis, age and diabetes mellitus were not found to be risk factors in the present study.

In conclusion, preoperative APRI was correlated with postoperative hepatic failure; in particular, APRI of ten or more carries a high risk of postoperative hepatic failure.

\section{References}

1. Lai EC, Fan ST, Lo CM, Chu KM, Liu CL, Wong J. Hepatic resection for hepatocellular carcinoma. An audit of 343 patients. Ann Surg. 1995;221:291-8.

2. Takenaka K, Kawahara N, Yamamoto K, Kajiyama K, Maeda T, Itasaka H, et al. Results of 280 liver resections for hepatocellular carcinoma. Arch Surg. 1996;131:71-6.

3. Wu CC, Ho WL, Yeh DC, Huang CR, Liu TJ, P'Eng FK. Hepatic resection of hepatocellular carcinoma in cirrhotic livers: is it unjustified in impaired liver function? Surgery. 1996;120:34-9.

4. Cohnert TU, Rau HG, Buttler E, Hernandez-Richter T, Sauter G, Reuter C, et al. Preoperative risk assessment of hepatic resection for malignant disease. World J Surg. 1997;21:396-400.

5. Lau H, Man K, Fan ST, Yu WC, Lo CM, Wong J. Evaluation of preoperative hepatic function in patients with hepatocellular carcinoma undergoing hepatectomy. Br J Surg. 1997;84:1255-9.

6. Lise M, Bacchetti S, Da Pian P, Nitti D, Pilati PL, Pigato P. Prognostic factors affecting long term outcome after liver resection for hepatocellular carcinoma: results in a series of 100 Italian patients. Cancer. 1998;82:1028-36.

7. Nonami T, Nakao A, Kurokawa T, Inagaki H, Matsushita Y, Sakamoto J, et al. Blood loss and ICG clearance as best prognostic markers of post-hepatectomy liver failure. Hepatogastroenterology. 1999;46:1669-72.

8. Pol B, Campan P, Hardwigsen J, Botti G, Pons J, Le Treut YP. Morbidity of major hepatic resections: a 100-case prospective study. Eur J Surg. 1999;165:446-53.

9. Farges O, Malassagne B, Flejou JF, Balzan S, Sauvanet A, Belghiti J. Risk of major liver resection in patients with underlying chronic liver disease: a reappraisal. Ann Surg. 1999;229: 210-5.

10. Yamanaka N, Okamoto E, Kuwata K, Tanaka N. A multiple regression equation for prediction of posthepatectomy liver failure. Ann Surg. 1984;200:658-63.

11. Didolkar MS, Fitzpatrick JL, Elias EG, Whitley N, Keramati B, Suter CM, et al. Risk factors before hepatectomy, hepatic function after hepatectomy and computed tomographic changes as indicators of mortality from hepatic failure. Surg Gynecol Obstet. 1989;169:17-26.

12. Noguchi T, Imai $T$, Mizumoto R. Preoperative estimation of surgical risk of hepatectomy in cirrhotic patients. Hepatogastroenterology. 1990;37:165-71.

13. Shimada M, Matsumata T, Akazawa K, Kamakura T, Itasaka H, Sugimachi K, et al. Estimation of risk of major complications after hepatic resection. Am J Surg. 1994;167:399-403.

14. Kwon AH, Ha-Kawa SK, Uetsuji S, Kamiyama Y, Tanaka Y. Use of technetium $99 \mathrm{~m}$ diethylenetriamine-pentaacetic acid-galactosyl-human serum albumin liver scintigraphy in the evaluation of preoperative and postoperative hepatic functional reserve for hepatectomy. Surgery. 1995;117:429-34.

15. Bruix J, Castells A, Bosch J, Feu F, Fuster J, Garcia-Pagan JC, et al. Surgical resection of hepatocellular carcinoma in cirrhotic patients: prognostic value of preoperative portal pressure. Gastroenterology. 1996;111:1018-22. 
16. Zimmermann H, Reichen J. Hepatectomy: preoperative analysis of hepatic function and postoperative liver failure. Dig Surg. 1998;15:1-11.

17. Kubota K, Makuuchi M, Kusaka K, Kobayashi T, Miki K, Hasegawa $\mathrm{K}$, et al. Measurement of liver volume and hepatic functional reserve as a guide to decision-making in resectional surgery for hepatic tumors. Hepatology. 1997;26:1176-81.

18. Miyagawa S, Makuuchi M, Kawasaki S, Kakazu T. Criteria for safe hepatic resection. Am J Surg. 1995;169:589-94.

19. Belghiti J, Ogata S. Assessment of hepatic reserve for indication of hepatic resection. J Hepatobiliary Pancreat Surg. 2005;12:1-3.

20. Nagasue N, Yukaya H, Ogawa Y, Kohno H, Nakamura T. Human liver regeneration after major hepatic resection. A study of normal liver and livers with chronic hepatitis and cirrhosis. Ann Surg. 1987;206:30-9.

21. Das BC, Isaji S, Kawarada Y. Analysis of 100 consecutive hepatectomies: risk factors in patients with liver cirrhosis or obstructive jaundice. World J Surg. 2001;25:266-72. discussion 272-273.

22. Wai CT, Greenson JK, Fontana RJ, Kalbfleisch JD, Marrero JA, Conjeevaram HS, et al. A simple noninvasive index can predict both significant fibrosis and cirrhosis in patients with chronic hepatitis C. Hepatology. 2003;38:518-26.

23. Castera L, Vergniol J, Foucher J, Le Bail B, Chanteloup E, Haaser M, et al. Prospective comparison of transient elastography, fibrotest, APRI, and liver biopsy for the assessment of fibrosis in chronic hepatitis C. Gastroenterology. 2005;128:34350 .

24. Horii K, Kubo S, Hirohashi K, Kinoshita H. Changes in erythrocyte deformability after liver resection for hepatocellular carcinoma associated with chronic liver disease. World J Surg. 1999;23:85-90.

25. Kubo S, Tsukamoto $T$, Hirohashi $K$, Tanaka $H$, Shuto $T$, Takemura $\mathrm{S}$, et al. Correlation between preoperative serum concentration of type IV collagen $7 \mathrm{~s}$ domain and hepatic failure following resection of hepatocellular carcinoma. Ann Surg. 2004; 239:186-93.

26. Liver Cancer Study Group of Japan. Classification of primary liver cancer. 1st English ed. Tokyo: Kanehara; 1997.

27. Edmondson HA, Steiner PE. Primary carcinoma of the liver: a study of 100 cases among 48, 900 necropsies. Cancer. 1954;7: 462-503.

28. Knodell RG, Ishak KG, Black WC, Chen TS, Craig R, Kaplowitz $\mathrm{N}$, et al. Formulation and application of a numerical scoring system for assessing histological activity in asymptomatic chronic active hepatitis. Hepatology. 1981;1:431-5.

29. Desmet VJ, Gerber M, Hoofnagle JH, Manns M, Scheuer PJ. Classification of chronic hepatitis: diagnosis, grading and staging. Hepatology. 1994;19:1513-20.

30. Liver Cancer Study Group of Japan. Primary liver cancer in Japan: clinicopathological features and results of surgical treatment. Ann Surg. 1990;211:277-87.
31. Nagasue N, Kohno H, Tachibana M, Yamanoi A, Ohmori H, ElAssal ON. Prognostic factors after hepatic resection for hepatocellular carcinoma associated with child-Turcotte class B and C cirrhosis. Ann Surg. 1999;229:84-90.

32. Takenaka K, Kanematsu T, Fukuzawa K, Sugimachi K. Can hepatic failure after surgery for hepatocellular carcinoma in cirrhotic patients be prevented? World J Surg. 1990;14:123-7.

33. Miyazaki S, Takasaki K, Yamamoto M, Tsugita M, Otsubo T. Liver regeneration and restoration of liver function after partial hepatectomy: the relation of fibrosis of the liver parenchyma. Hepatogastroenterology. 1999;46:2919-24.

34. Tanaka S, Kubo S, Tsukamoto T, Hirohashi K, Tanaka H, Shuto $\mathrm{T}$, et al. Risk factors for intractable pleural effusion after liver resection. Osaka City Med J. 2004;50:9-18.

35. Eguchi H, Umeshita K, Sakon M, Nagano H, Ito Y, Kishimoto SI, et al. Presence of active hepatitis associated with liver cirrhosis is a risk factor for mortality caused by posthepatectomy liver failure. Dig Dis Sci. 2000;45:1383-8.

36. Fan ST, Lai EC, Lo CM, Ng IO, Wong J. Hospital mortality of major hepatectomy for hepatocellular carcinoma associated with cirrhosis. Arch Surg. 1995;130:198-203.

37. Matsumata T, Kanematsu T, Yoshida Y, Furuta T, Yanaga K, Sugimachi K. The indocyanine green test enables prediction of postoperative complications after hepatic resection. World J Surg. 1987;11:678-81.

38. Fujio N, Sakai K, Kinoshita H, Hirohashi K, Kubo S, Iwasa R, et al. Results of treatment of patients with hepatocellular carcinoma with severe cirrhosis of the liver. World J Surg. 1989;13:211-7. discussion 217-218.

39. Hemming AW, Scudamore CH, Shackleton CR, Pudek M, Erb $\mathrm{SR}$. Indocyanine green clearance as a predictor of successful hepatic resection in cirrhotic patients. Am J Surg. 1992;163:5158.

40. Yasui M, Harada A, Torii A, Nakao A, Nonami T, Takagi H. Impaired liver function and long-term prognosis after hepatectomy for hepatocellular carcinoma. World J Surg. 1995;19:43943.

41. Midorikawa Y, Kubota K, Takayama T, Toyoda H, Ijichi M, Torzilli G, et al. A comparative study of postoperative complications after hepatectomy in patients with and without chronic liver disease. Surgery. 1999;126:484-91.

42. Imamura H, Sano K, Sugawara Y, Kokudo N, Makuuchi M. Assessment of hepatic reserve for indication of hepatic resection: decision tree incorporating indocyanine green test. J Hepatobiliary Pancreat Surg. 2005;12:16-22.

43. Poon RT, Fan ST, Tsang FH, Wong J. Locoregional therapies for hepatocellular carcinoma: a critical review from the surgeon's perspective. Ann Surg. 2002;235:466-86. 